\title{
Ordnung ins Chaos bringen
}

Interview: Bruno Kesseli

Dr. med. et lic. phil., Chefredaktor

\begin{abstract}
Gegensätze ziehen sich an der Fortbildungstagung des Kollegiums für Hausarztmedizin (KHM) seit Jahren an und bilden als Kongressthemen spannungsvolle Paare. Dies ist auch bei der 17. Auflage nicht anders, die am 25./26. Juni einmal mehr in Luzern stattfindet und unter dem Motto "Ordnung \& Chaos» steht. Im Interview äussert sich Franziska Zogg, Präsidentin des Tagungskomitees, zu den Charakteristika des Kongresses.
\end{abstract}

\begin{abstract}
Mit "Ordnung und Chaos» wurde für die 17. Auflage des KHM-Kongresses einmal mehr ein originelles Motto gefunden. Wie kommen die Tagungsthemen zustande?

Ausschlaggebend ist der übergeordnete Zyklus «Gegensätze». So waren frühere Tagungsthemen unter anderem «gut und böse», «jung und alt» oder «feucht und
\end{abstract}

"Ich hoffe, dass viele interessante und kritische Anregungen auch aus dem Publikum kommen.»

trocken». Wir lassen unseren Assoziationen im Komitee freien Lauf und entscheiden uns jeweils für das Thema, das uns selbst am meisten fasziniert. «Ordnung und Chaos» bietet ein breites Spektrum von ganz unterschiedlichen Fragestellungen und war deshalb diesmal unbestrittener Favorit.

\section{Die Hauptreferate sind in diesem Jahr den Themen Psychose, Immunsystem, Kommunikation und Hormone gewidmet. Nach welchen Kriterien wurden diese Gebiete ausgewählt? \\ Sie beinhalten selbst oft Chaos oder sind schlecht über- schaubar und verlangen besonders nach Ordnung - zumindest im Kopf der behandelnden Ärzte.}

\section{Der Praxisbezug wird am KHM-Kongress gross-} geschrieben. Wie gewährleisten Sie ihn?

Alle unsere Referate und Seminare werden von zwei Personen gemeinsam gehalten respektive geleitet: von einer Fachexpertin zusammen mit einer Kinder- oder

«Damit können wir aktuelles Fachwissen bieten, das für die tägliche Praxis relevant ist.»

Hausärztin aus der Praxis. Damit können wir aktuelles Fachwissen bieten, das für die tägliche Praxis relevant ist.

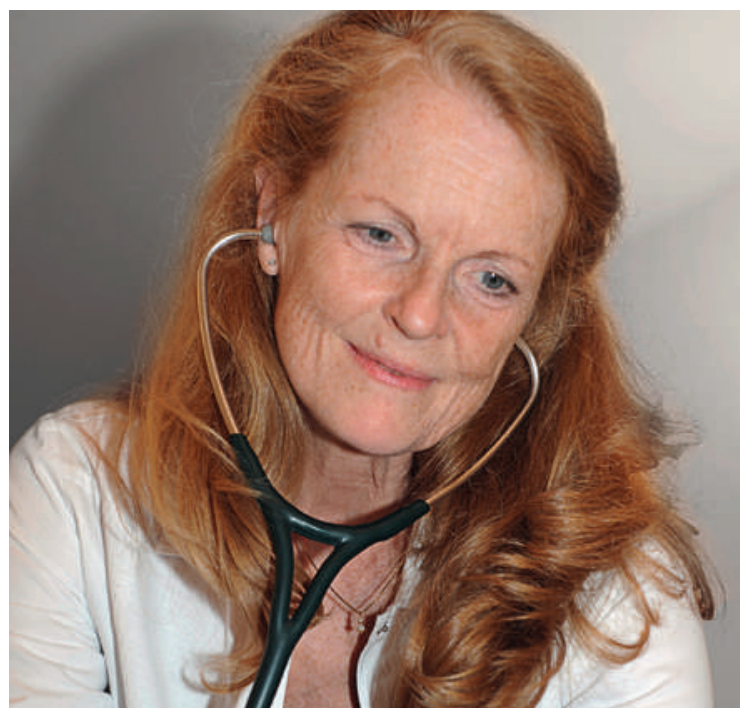

Auch die Tagungspräsidentin gewährleistet den Praxisbezug: Franziska Zogg ist als Hausärztin tätig.

Auf reges Interesse stösst jeweils der standespolitische Roundtable. Was darf das Publikum diesmal erwarten? Auch hier liessen wir uns vom "Chaos" und dem Wunsch nach Ordnung leiten: Social media und Internet haben unterschiedliche Auswirkungen auf die ArztPatienten-Beziehung und sind in ihrer Entwicklung nicht einfach überblickbar. Ich bin gespannt, welche Dimensionen uns die Experten aufzeigen können und hoffe, dass viele interessante und kritische Anregungen auch aus dem Publikum kommen.

\section{Zur Person}

Franziska Zogg ist Fachärztin für Allgemeine Innere Medizin und als Hausärztin in Zug tätig. Seit vielen Jahren ist sie in der Standespolitik aktiv, aktuell unter anderem als Vorstandsmitglied der Schweizerischen Gesellschaft für Allgemeinmedizin SGAM und Vizepräsidentin der Ärztegesellschaft des Kantons Zug. An der diesjährigen Fortbildungstagung des KHM präsidiert sie das Organisationskomitee. 


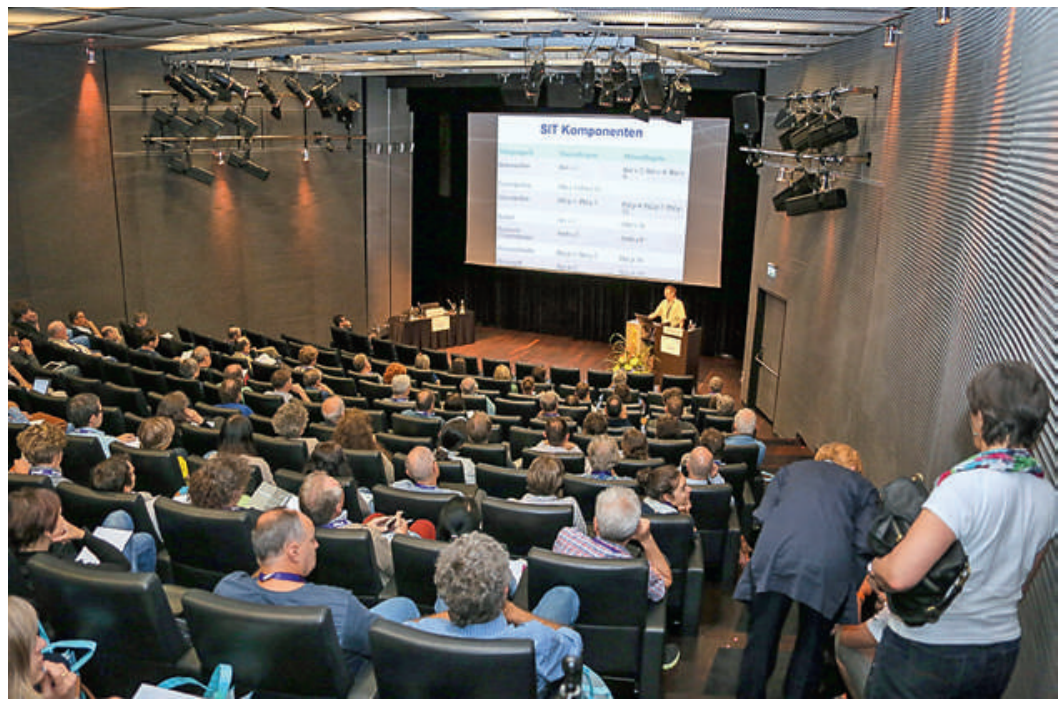

Volle Säle: Der KHM-Kongress erfreut sich seit Jahren grosser Beliebtheit.

Am Kongress findet traditionellerweise die Generalversammlung von "Hausärzte Schweiz» statt. Trügt der Eindruck, dass das Interesse der Mitglieder an diesem Anlass in den letzten Jahren nachgelassen hat? Ist der Verband ein Opfer seines eigenen Erfolgs? Die Beteiligung an der GV von Hausärzte Schweiz ist konstant niedrig - aber wohl eher Ausdruck der andersartigen Organisation: Die richtungsweisenden Entscheide für den Verband finden an der Delegiertenversammlung und nicht an der GV statt. Dort wird in erster Linie einfach informiert.

«Wir wollen ganz einfach attraktiv bleiben. Das bedeutet, das Konzept und die Wahl der Themen und Referentinnen dauernd zu überdenken.»

Der KHM-Kongress erfreut sich seit Jahren grosser Beliebtheit bei den Hausärztinnen und -ärzten.

Was ist sein Erfolgsgeheimnis?

Mehr zum diesjährigen KHM-Kongress unter www.khm-kongress.ch/ khm2015

bkesseli[at]emh.ch
Wenn Sie das Kongresskonzept kritisch anschauen: Wo sehen Sie Schwachpunkte oder Verbesserungspotential?

Wir wollen ganz einfach attraktiv bleiben. Das bedeutet, das Konzept und die Wahl der Themen und Referentinnen dauernd zu überdenken. Dabei sind uns die Rückmeldungen der Teilnehmer zum Kongress eine grosse Hilfe.

Die Fusion von SGAM und SGIM zur SGAIM steht bevor. Wird sie sich auf den KHM-Kongress auswirken?

Die Neugründung der SGAIM hat keinen Einfluss auf den KHM-Kongress. Die Schweizerische Gesellschaft für Pädiatrie ist der dritte wichtige Partner in der KHMStiftung und wird dies auch nach der Fusion bleiben. Sie ist mit dem Modul Pädiatrie übrigens auch immer präsent am Kongress.

\section{Zum Schluss: Welche Veranstaltung an diesem} Kongress sollte man auf keinen Fall verpassen? Das Intermezzo sorpresa - wir können uns hingeben und müssen dabei nicht einmal auf eine andere interessante Fortbildung verzichten.

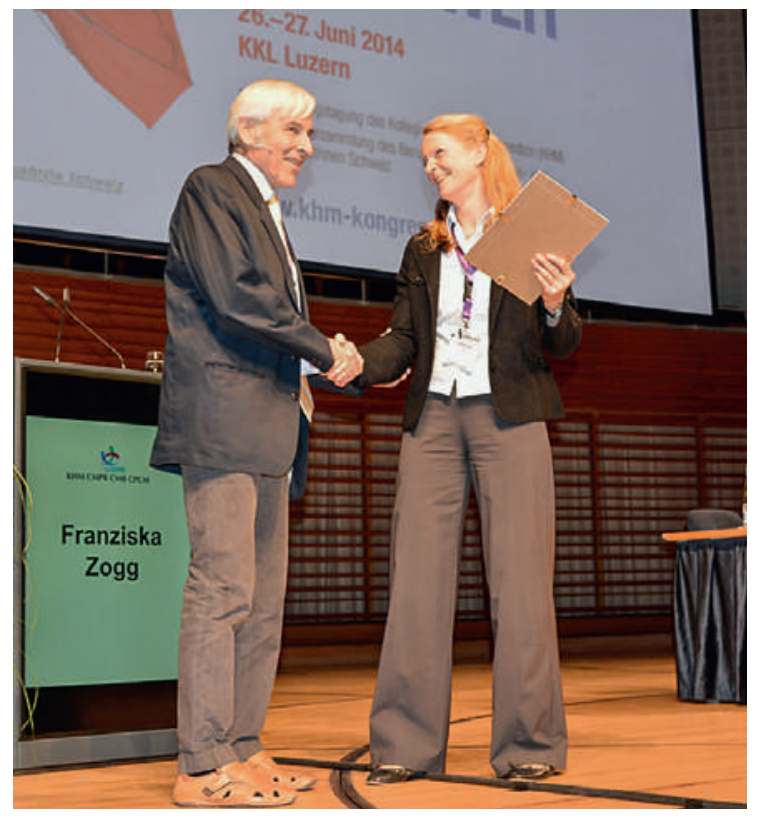

Franziska Zogg mit Professor Peter Tschudi, der 2014 als "KHM-Kopf des Jahres» ausgezeichnet wurde. 